\title{
Theory of mind and psychopathy: can psychopathic individuals read the 'language of the eyes'?
}

\author{
R.A. Richell ${ }^{\text {b }}$, D.G.V. Mitchell ${ }^{\mathrm{a}, \mathrm{b}}$, C. Newman ${ }^{\mathrm{b}}$, A. Leonard $^{\mathrm{c}}$, \\ S. Baron-Cohen ${ }^{\text {d }}$, R.J.R. Blair ${ }^{\text {a,* }}$ \\ ${ }^{a}$ Unit on Affective Cognitive Neuroscience, Mood and Anxiety Disorders Program, National Institute of Mental Health, \\ 15 K North Drive, Room 206, MSC 2670 Bethesda, MD 20892-2670, USA \\ ${ }^{\mathrm{b}}$ Institute of Cognitive Neuroscience, Department of Psychology, University College, London, UK \\ ${ }^{\mathrm{c}}$ HMP Wormwood Scrubs, London, UK \\ ${ }^{\mathrm{d}}$ Autism Research Centre, Departments of Experimental Psychology and Psychiatry, University of Cambridge, Cambridge, UK
}

Received 14 August 2001; received in revised form 10 July 2002; accepted 2 September 2002

\begin{abstract}
There have been suggestions that Theory of Mind (ToM) impairment might lead to aggressive behaviour and psychopathy. Psychopathic and matched non-psychopathic individuals, as defined by the Hare Psychopathy Checklist [The Hare Psychopath Checklist-Revised, 1991] completed the 'Reading the Mind in the Eyes' ToM Test [Journal of Child Psychology and Psychiatry, 1997;38:813]. This test requires the self-paced identification of mental states from photographs of the eye region alone. Results indicated that the psychopathic individuals did not present with any generalised impairment in ToM. The data are discussed with reference to the putative neural system mediating performance on this task and models of psychopathy.
\end{abstract}

(C) 2002 Elsevier Science Ltd. All rights reserved.

Keywords: Psychopathy; Theory of mind; Amygdala

\section{Introduction}

Psychopathy is a disorder characterised in part by callousness, a diminished capacity for remorse, superficial charm, impulsivity, and poor behavioural controls. The disorder is identified using a clinically-based rating scale, the Psychopathy Checklist-Revised, which has been extensively validated (PCL-R; [9]).

There have been repeated suggestions that a deficient or a biased understanding of other people's mental states (i.e. impaired Theory of Mind (ToM)) might lead to antisocial and aggressive behaviour and psychopathy (e.g. $[7,8])$. Thus, Feshbach [8] has argued that role-taking (which involves the representation of another individual's mental states) is a prerequisite for empathic responding which, in turn, is involved in the inhibition of antisocial behaviour. Individuals deficient in role-taking should be less likely to empathize and thus less likely to inhibit antisocial behavior.

The data has been inconsistent, however, Hughes et al. [10] did find some indication of ToM impairment in their

\footnotetext{
* Corresponding author.

E-mail address: blairj@intra.nimh.nih.gov (R.J.R. Blair).
}

"hard-to-manage" preschoolers relative to the comparison group. However, two out of three studies on adult psychopathic individuals found no indications of ToM impairment ([6,15] did not, [16] did). Of course, the failure to find group differences in the studies with adults may reflect the ease of many ToM measures. The development of an advanced, adult ToM Task has attempted to overcome this problem $[1,3]$. This test, named the 'Reading the Mind in the Eyes' Test, requires participants to view photographs of the eye region of individuals, and from this information alone to attribute a mental state to the person in the photograph (forced choice from a selection of mental state descriptors). Individuals with autism, with known ToM deficits, present with significant difficulty on this task [3].

The Eyes Test is also interesting because of its known anatomical correlates. Thus, a recent functional Magnetic Resonance Imaging study showed that presentation of the task produced increased activation in several areas, including the dorsolateral prefrontal and the left medial frontal cortices, the superior temporal gyrus, and the left amygdala. The finding of amygdala activation is of particular interest given the extensive literature suggesting amygdala dysfunction in psychopathy (see, for a review, [5]). Thus, if the Eyes Test 
requires the involvement of the amygdala, and psychopathy reflects amygdala dysfunction, it should be predicted that individuals with psychopathy will perform poorly on the Eyes Test.

\section{Methods}

\subsection{Participants}

From a pool of 200 men incarcerated in one of three forensic institutions in the London area, two groups of male participants were identified who satisfied the criteria for the psychopathic $(n=19)$ and non-psychopathic control ( $n=18$ ) groups. In accordance with the literature and the guidelines of the PCL-R [10], individuals with a score of 30 or above on the PCL-R were assigned to the psychopathic group, whilst those with a score of 20 or less were assigned to the control group. The ages of the participants ranged from 21 to 44 years, with a mean of 32.7 years (S.D. $=7.4$ ). The mean ages of the psychopathic and control groups were 32.2 (S.D. $=6.8$ ) and 33.3 (S.D. $=8.1$ ) years, respectively.

The Raven's Advanced Progressive Matrices [12] was administered to provide an estimate of intelligence. The Raven's Scores of the participants ranged from 4 to 12, with a mean of 8.5 (S.D. $=2.3$ ). The mean Raven's Scores of the psychopathic and control groups were 8.6 (S.D. $=2.3$ ) and 8.4 (S.D. $=2.4$ ), respectively. Participant characteristics are given in Table 1 . The two groups did not differ significantly in terms of age or Raven's score. Written consent was obtained from each inmate who participated in the study, and all were informed that they were free to withdraw from the study at any time.

\subsection{Psychopathy checklist-revised (PCL-R)}

The PCL-R [9] consists of 20 behavioural items that are scored on the basis of a file review and, where possible, a semi-structured interview. Each item has a maximum possible score of 2, and the maximum total score is therefore 40. The PCL-R has been shown to be a valid and reliable method for assessing psychopathy [10].

Inmates were scored by two independent raters. Inter-rater reliability, assessed by Spearman Rank correlation, was high $\left(r_{\text {ranks }}=0.96, P<0.001\right)$. The agreement between the two raters for diagnostic group (psychopathic versus control)

Table 1

Participant characteristics and mean score of items correctly identified on the 'Reading the Mind in the Eyes' Test (S.D. in brackets)

\begin{tabular}{lllll}
\hline Group & PCL-R & Age & Raven's & $\begin{array}{l}\text { Eyes test } \\
\text { score }\end{array}$ \\
\hline $\begin{array}{l}\text { Psychopathic inmates } \\
(n=19)\end{array}$ & $33.3(2.2)$ & $32.2(6.8)$ & $8.6(2.3)$ & $23.9(5.3)$ \\
$\begin{array}{l}\text { Controls }(n=18) \\
{ }^{*} P<0.001, \text { significantly different from psychopathic group. }\end{array}$
\end{tabular}

was $100 \%$. The mean PCL-R scores of the psychopathic and control groups are given in Table 1.

\subsection{Reading the mind in the eyes task (revised version)}

The revised version of the 'Reading the Mind in the Eyes' Test was administered [3]. In brief, participants were presented with a series of 40 photographs of the eye region of the face of different actors and actresses. An equal number of male and female faces were presented. Four complex mental state descriptors, e.g. dispirited, bored, were printed around the photo, one at each corner. One of these words (the target word) correctly identified the mental state of the person in the photo, whilst the others were included as foils. Targets and foils were piloted by the original authors of the task, and where possible the three foil words have the same emotional valence as the target word [3]. Participants were instructed to choose which of the four words best described what the person in the photo was thinking or feeling. Participants were asked to read all four words before making their choice and, if they felt that more than one word was applicable, to "choose just one word, the word which you consider to be most suitable'. This task is considered an advanced ToM test as participants are required to attempt to put themselves in to the mind of the person shown in the photograph, and attribute a relevant mental state to them.

The task was self-paced, i.e. participants could take as long as they wanted to determine the mental state of the person in each photograph, and progressed to the next photograph when ready. Participants were also provided with a glossary of all the mental state terms used in the task, and were told that they could consult the glossary at any point if they were unsure of the definition of any descriptor term used. The test was scored by totalling the number of items (photographs) correctly identified by the participant, i.e. the number of mental states correctly identified. In accordance with Baron-Cohen et al. [3], four items from the test were excluded as their normative data showed that $<50 \%$ of the general population examined selected the correct target descriptor. The maximum total score on the test was therefore 36.

\subsection{Procedure}

All participants were tested individually, in a quiet room attached to the wing. The revised version of the 'Reading the Mind in the Eyes' Test and the Raven's Advanced Progressive Matrices were administered as part of a larger neuropsychological test battery. Verbal instructions were given to each participant prior to commencing the test.

\section{Results}

The mean total number of items correctly identified in the test was calculated for the psychopathic and control 
groups. These means, and their standard deviations, are presented in Table 1. Although the control group had a slightly higher total score than the psychopathic group, an independent sample's $t$-test showed that there was no significant difference between the groups in the total number of items correctly identified $(t=1.49, P=0.14)$. There were no significant correlations between age, or Raven's score, and performance on the Reading the Mind in the Eyes test ( $r=$ 0.03 and 0.17 ).

The group of subjects with high-functioning autism or Asperger's syndrome and the general population control group tested by Baron-Cohen et al. [3] had a mean total score of 21.9 (S.D. $=6.6$ ) and 26.2 (S.D. = 3.6), respectively, on the Eyes Test. Comparison of the scores produced in this study with this autistic group showed that $17 \%$ of the control participants, but $42 \%$ of the psychopathic participants, had a total score at or below the mean value of this autistic group. However, the percentage of non-psychopathic versus psychopathic participants scoring below the mean value of the autistic group was not significantly different $\left(\chi^{2}(1)=2.86\right.$; NS). The mean total score of the control group used in this study (26.3, S.D. $=4.3)$ was very similar to that of the general population controls tested by Baron-Cohen et al. [3]. Moreover, $61 \%$ of the control group and $47 \%$ of the psychopathic group, scored at or above the mean value of Baron-Cohen et al. general population mean; these percentages were again not significantly different $\left(\chi^{2}(1)=0.70\right.$; NS $)$.

\section{Discussion}

This study investigated the performance of psychopathic and non-psychopathic individuals on an adult, advanced ToM test. The results showed that both groups performed equally well on the test overall, indicating that psychopathic individuals present with no generalised impairment in ToM. Of the three previous studies of ToM in psychopathic individuals, two found no indications of ToM impairment $[6,15]$. Thus, the results of this current study, further confirm the absence of any ToM impairment in psychopathic individuals and do so using a task considered to be considerably more sensitive than those previously used [3].

There is abundant data indicating impairment in psychopathic individuals in processing facial affect; in particular, the expressions of fear and sadness [4]. Indeed, the fact that the expression recognition impairment is selective for fearful and sad expressions supports the amygdala dysfunction hypothesis of psychopathy (see, for a review, [5]). This is interesting given recent neuro-imaging findings study revealing amygdala activation during performance of the Eyes Test [2]. If psychopathic individuals present with amygdala dysfunction and if appropriate amygdala functioning is necessary for successful performance on the Eyes task, psychopathic individuals should have performed poorly on the Eyes task. Yet they did not, this could be taken as challeng- ing for the amgydala dysfunction hypothesis of psychopathy. However, there is both considerable neuropsychological and neuro-imaging data in support of this hypothesis. Thus, psychopathic individuals perform poorly on tasks requiring both the basolateral and central nucleus of the amygdala (e.g. recognition of fearful expressions, instrumental learning, aversive conditioning and potentiation of startle reflex; see, for a review, [5]). Moreover, recent neuro-imaging data has shown reduced amygdala volume in psychopathic individuals [14] and reduced amygdala activation in psychopathic individuals during an emotional memory task [11].

Alternatively, it could be argued that the amygdala activation during performance of the Eyes task [2] reflects its' interconnections with other brain regions. These other brain areas may be necessary for successful performance on the task and because of their association with the amygdala result in amygdala activation even if this activation is not a prerequisite for successful task performance. However, recent data suggests that patients with amygdala lesions perform poorly on an earlier form of the Eyes task [13]. This suggests that the amygdala does play a role in mediating performance on this task in healthy individuals.

A second alternative is that intact amygdala functioning is required for successful performance on the Eyes task in healthy individuals. However, in a population such as psychopathic individuals where there may be early reduced, but not absent, amygdala functioning [14], other, perhaps cortical, regions may compensate during development for this reduced amygdala functioning. Interestingly, the selective impairment shown by psychopathic individuals in the processing of facial expressions is far more marked in children with psychopathic tendencies than in adult psychopathic individuals (see, for a review, [5]). This suggests compensation during development for the early impairment. If this possibility is correct, we can assume that in a neuro-imaging study, psychopathic individuals would show reduced amygdala activation whilst performing the Eyes task even though, as this study shows, there would be no impairment in their behavioural performance. The argument would be that, unlike individuals with Asperger's syndrome [3], Theory of Mind is intact in psychopathic individuals even if the neural architecture mediating Theory of Mind is not equivalent to that used by healthy developing populations.

In summary, this study indicates that psychopathic individuals do not present with a generalised impairment in ToM. Following on from earlier work demonstrating that psychopathic individuals present with no impairment in correctly attributing mental states to protagonists in verbal narratives, the current study shows that they present with no difficulty attributing mental states to others on the basis of information of only their eyes.

\section{Acknowledgements}

This work was supported by the Medical Research Council (ref. G9716841) and Department of Health (VISPED 
initiative). The authors would like to thank the Psychology Department and staff at HMP Wormwood Scrubs.

\section{References}

[1] Baron-Cohen S, Jolliffe T, Mortimore C, Robertson M. Another advanced test of theory of mind: evidence from very high functioning adults with autism or asperger syndrome. Journal of Child Psychology and Psychiatry 1997;38:813-22.

[2] Baron-Cohen S, Ring HA, Wheelwright S, Bullmore ET, Brammer MJ, Simmons A, et al. Social intelligence in the normal and autistic brain: an fMRI study. European Journal of Neuroscience 1999;11:1891-8.

[3] Baron-Cohen S, Wheelwright S, Hill J, Raste Y, Plumb I. The "Reading the Mind in the Eyes" test revised version: a study with normal adults, and adults with Asperger syndrome or high-functioning autism. Journal of Child Psychology and Psychiatry 2001;42:241-51.

[4] Blair RJ, Jones L, Clark F, Smith M. The psychopathic individual: a lack of responsiveness to distress cues? Psychophysiology 1997;34: 192-8.

[5] Blair RJ. Neuro-cognitive models of aggression. Journal of Neurology, Neurosurgery, and Psychiatry 2001;71:727-31.

[6] Blair RJ, Sellars C, Strickland I, Clark F, Williams A, Smith M, et al. Theory of mind in the psychopath. Journal of Forensic Psychiatry 1996;7:15-25.

[7] Crick NR, Dodge KA. A review and reformulation of social information-processing mechanisms in children's social adjustment. Psychological Bulletin 1994;115:74-101.

[8] Feshbach ND. Parental empathy and child adjustment/maladjustment. In: Eisenberg N, Strayer J, editors. Empathy and its development. New York: Cambridge University Press; 1987. p. 271-91.

[9] Hare RD. The Hare Psychopathy Checklist-Revised. Toronto (Ont.): Multi-Health Systems; 1991.

[10] Hughes C, Dunn J, White A. Trick or treat? Uneven understanding of mind and emotion and executive dysfunction in "hard-tomanage" preschoolers. Journal of Child Psychology and Psychiatry 1998;39:981-94.

[11] Kiehl KA, Smith AM, Hare RD, Mendrek A, Forster BB, Brink $\mathrm{J}$, et al. Limbic abnormalities in affective processing by criminal psychopaths as revealed by functional magnetic resonance imaging. Biological Psychiatry 2001;50:677-84.

[12] Raven JC. Advanced progressive matrices, Set I. Oxford: Oxford Psychologists Press; 1976.

[13] Stone VE, The role of the frontal lobes and the amygdala in theory of mind. In: Baron-Cohen S, Tager-Flusberg H, Cohen D, editors. Understanding other minds: perspectives from autism and cognitive neuroscience. Oxford: Oxford University Press, 1999.

[14] Tiihonen J, Hodgins S, Vaurio O, Laakso M, Repo E, Soininen H, et al. Amygdaloid volume loss in psychopathy. Society for Neuroscience Abstracts 2000;2017.

[15] Widom CS. An empirical classification of female offenders. Criminal Justice and Behaviour 1978;5:35-52.

[16] Widom CS. Interpersonal and personal construct systems in psychopaths. Journal of Consulting and Clinical Psychology 1976;44: 614-23. 